lustitia Socialis. Revista Arbitrada de Ciencias Jurídicas.

Año V. Vol. V. №3. Edición Especial. 2020-III:

Universidad Regional Autónoma de los Andes

Hecho el depósito de Ley: FA2016000064

ISSN: 2542-3371

FUNDACIÓN KOINONIA (F.K). Santa Ana de Coro, Venezuela

Julián Rodolfo Santillán-Andrade; Jaritza Andreina Aguirre-Aguilar

http://dx.doi.org/10.35381/racii.v5i3.1075

\title{
La responsabilidad del Estado como obligado subsidiario en los juicios de fijación de pensiones alimenticias
}

\section{State liability as a subsidiary liability in food pension fixing trials}

Julián Rodolfo Santillán-Andrade

us.juliansantillan@uniandes.edu.ec

Universidad Regional Autónoma de los Andes, Santo Domingo

Ecuador

https://orcid.org/0000-0002-2614-5676

Jaritza Andreina Aguirre-Aguilar

jary 1996 @hotmail.com

Universidad Regional Autónoma de los Andes, Santo Domingo

Ecuador

Recibido: 31 de octubre de 2020

Revisado: 10 de octubre de 2020

Aprobado: 05 de diciembre de 2020

Publicado: 10 de diciembre de 2020 


\title{
RESUMEN
}

La investigación tiene como objetivo es realizar un análisis jurídico sobre la responsabilidad del Estado ecuatoriano como obligado subsidiario en los juicios de fijación de pensión alimentaria, a efecto de no vulnerar los derechos de los niños, niñas y adolescente, que se ven afectados por las malas praxis en la materia de amparo legal. Desde un enfoque cuantitativo de tipo descriptivo de campo, no experimental. Metodológicamente bajo un enfoque analítico-sintético y a nivel descriptivo documental desde un diseño bibliográfico. Se construyó y aplicó una encuesta a 30 profesionales del derecho, quienes respondieron a una serie de interrogantes, previamente sometidas a juicio de expertos. Analizándose las respuestas y contrastando con los aportes teóricos. Se concluye que no existen normas legalmente relacionadas al tema tratado; es necesario promover reformas donde normalicen la participación del Estado como subsidiario por excepción en pensión alimentaria, amparado en el interés superior de la niña, niño y adolescente.

Descriptores: Derechos humanos; responsabilidad del Estado; mediación. (Palabras tomadas del Tesauro UNESCO).

\begin{abstract}
The objective of the investigation is to carry out a legal analysis on the responsibility of the Ecuadorian State as an obligated subsidiary in the lawsuits for setting alimony, in order not to violate the rights of children and adolescents, who are affected by the bad practice in the matter of legal protection. From a quantitative, descriptive field approach, not experimental. Methodologically under an analytical-synthetic approach and at a documentary descriptive level from a bibliographic design. A survey was constructed and applied to 30 legal professionals, who responded to a series of questions, previously submitted to expert judgment. Analyzing the answers and contrasting with the theoretical contributions. It is concluded that there are no regulations legally related to the subject matter; It is necessary to promote reforms where they normalize the participation of the State as subsidiary by exception in alimony, protected in the best interests of the girl, boy and adolescent.
\end{abstract}

Descriptors: Human rights; responsibility of the State; mediation (Words taken from the UNESCO Thesaurus). 


\section{INTRODUCCIÓN}

El derecho a percibir alimentos que tienen en Ecuador los niños/as y adolescentes, ha vivido un proceso evolutivo relevante para llegar a constituirse como tal, mismo derecho que se encuentra reconocido en el Código de la Niñez y Adolescencia, Derecho de Alimentos (2003) capítulo I Articulo (2) disponiendo que se dé un tratamiento especial que brinde las debidas garantías al cumplimiento del derecho antes referido; el derecho a alimentos es connatural a la relación parentofilial y está relacionado con el derecho a la vida, la supervivencia y una vida digna. Implica la garantía de proporcionar los recursos necesarios para la satisfacción de las necesidades básicas de los alimentarios que incluye:

1. Alimentación nutritiva, equilibrada y suficiente;

2. Salud integral: prevención, atención médica y provisión de medicinas;

3. Educación;

4. Cuidado;

5. Vestuario adecuado;

6. Vivienda segura, higiénica y dotada de los servicios básicos;

7. Transporte;

8. Cultura, recreación y deportes; y,

9. Rehabilitación y ayudas técnicas si el derechohabiente tuviere alguna discapacidad

En concordancia el Código Civil en su Título XV se refiere a los alimentos, como se dividen y que por ley les corresponde a ciertas personas, en concreto los siguientes artículos especifican ciertas peculiaridades:

Art..368.- Las reglas generales a que está sujeta la prestación de alimentos, son las siguientes, sin perjuicio de las disposiciones especiales que contiene este Código, respecto de ciertas personas.

Art.369.- Los alimentos se dividen en congruos y necesarios.

Congruos, son los que habilitan al alimentado para subsistir modestamente, de un modo correspondiente a su posición social. Necesarios, los que le dan lo que basta para sustentar la vida.

Los alimentos, sean congruos o necesarios, comprenden la obligación de proporcionar al alimentario menor de dieciocho años, cuando menos, la enseñanza primaria. 
Es así que se relaciona la necesidad de contar con una administración de justicia especializada, lo que lleva al análisis del contenido de la Ley Reformatoria al Título V, Libro II del Código Orgánico de la Niñez y Adolescencia, dentro de esta materia, en razón de que a pesar que se han desplegado arduos esfuerzos, por desarrollarla y disponer que su cumplimiento y vigencia sean eficaces, ha propiciado que derechos de obligados subsidiarios sean vulnerados, retrasados y no les estén dando el debido tramite que se requiere; es por esa razón que se cree que el Estado debería ser el principal obligado subsidiario de prestar alimentos en caso de que los que menciona el Código antes referido no puedan cumplir con la obligación. En tal sentido Beloff (2007) afirma:

El enfoque esquizofrénico de la compasión-represión perdura todavía hoy en muchas cabezas y en algunas pocas leyes; de lo que no cabe duda en cambio es de que el presente y el futuro de la infancia ya son una cuestión de justicia. (p.46)

El estado ecuatoriano, en cumplimiento a los diferentes tratados internacionales dispone dentro de sus políticas de estado la promoción y protección de los ciudadanos, garantizando el debido proceso. Ahora bien, el legislador ha establecido el derecho de alimentos, y ha previsto el mecanismo cuando conste incumplimiento en el pago de pensiones alimenticias por parte del alimentante y lo ha regulado en el Código Orgánico General de Procesos (COGEP), en el Art. 137 que establece: si la parte actora dentro del proceso iniciado, solicita una liquidación por concepto de pensiones alimenticias atrasadas y en la mismas se constata el incumplimiento por parte del obligado de dos o más pensiones alimenticias, el juzgador a petición de parte dispondrá la prohibición de salida del país del alimentante y convocará audiencia de formulación de pago en un término máximo de diez días (Asamblea Nacional del Ecuador, 2015).

Al respecto Cedeño (2019), indica la intervención del Estado:

La participación jurídica del Estado en la familia debe tener por propósito garantizar los derechos de sus integrantes a efecto de que no sean restringidos de una manera arbitraria, ni se lesione su esencia al ser el núcleo fundamental de la sociedad, por la coexistencia con otros derechos 
lustitia Socialis. Revista Arbitrada de Ciencias Jurídicas.

Año V. Vol. V. №3. Edición Especial. 2020-III:

Universidad Regional Autónoma de los Andes

Hecho el depósito de Ley: FA2016000064

ISSN: 2542-3371

FUNDACIÓN KOINONIA (F.K). Santa Ana de Coro, Venezuela

Julián Rodolfo Santillán-Andrade; Jaritza Andreina Aguirre-Aguilar

y deberes, salvo que se trate del interés superior de alguno de sus miembros. (p.195)

En este mismo orden de idea el artículo 5 de la Ley Reformatoria al Código Orgánico de la Niñez y Adolescencia establece: obligados a la prestación de alimentos. Los padres son los titulares principales de la obligación alimentaria, aun en los casos de limitación, suspensión o probación de la patria potestad. En casos de audiencia, impedimento, insuficiencia de recursos o discapacidad de los obligados principales, debidamente comprobado por quien lo alega, la autoridad competente ordenara que la prestación de alimentos sea pagada o completada por uno o más de los siguientes obligados subsidiarios, en atención a su capacidad económica, siempre y cuando no se encuentre discapacitados, en su orden: 1) Los abuelos/as; 2. Los hermanos/as que hayan cumplido 21 años y no estén comprendidos en los casos de los numerales dos y tres del artículo anterior; y, 3. Los tios/as. La autoridad competente, en base al orden previsto en los numerales precedentes, en los grados de parentesco señalados, de modo simultáneo y con base en sus recursos, regulara la proporción en la que dichos parientes proveerán la pensión alimenticia, hasta completar el monto total de la pensión fijada o asumirla en su totalidad, según el caso. Los parientes que hubieren realizado el pago podrán ejercer la acción de repetición de lo pagado contra el padre y/o la madre. los jueces aplicaran de oficio los instrumentos internacionales ratificados por el Ecuador a fin de garantizar el derecho de alimentos de los niños, niñas y adolescentes, hijas e hijos de padres o madres que hubieran migrado al exterior, y dispondrán todas las medidas necesarias para asegurar el cobro efectivo de la pensión. La autoridad central actuará con diligencia para asegurar el respeto de los derechos de los niños, niñas y adolescentes, y, responderá en caso de negligencia. (Código de la Niñez y Adolescencia, 2017).

La obligación que tienen los padres sobre prestar alimentos según la norma referida, recae sobre los subsidiarios, es decir, sobre abuelos, hermanos o tíos respectivamente y solo es exigible siempre y cuando el obligado principal no esté en condiciones de prestarlo, esto debidamente comprobado. De lo dicho; la obligación subsidiaria es condicional y está sujeta a una situación condicional, es decir que si se 
demuestra que el obligado principal no se halla en reales imposibilidades de cumplir con la demanda alimentaria, la obligación inmediata le regresara, así como por ejemplo cuando el deudor principal argumentado incapacidad económica sin justificación previa, la ley le demandara el cumplimiento de la responsabilidad, aun cuando ya haya sido endosada dicha obligación a los subsidiarios.

Si bien es cierto la norma suprema del Ecuador, la Constitución de la Republica enfatiza en el artículo 44 lo siguiente: El Estado, la sociedad y la familia promoverán de forma prioritaria el desarrollo integral de las niñas, niños y adolescentes, y aseguraran el ejercicio pleno de sus derechos, se atenderán al principio de su interés superior y sus derechos prevalecerán sobre lo de las demás persona. (Asamblea Constituyente, 2008). Es decir que el Ecuador reconoce de manera tácita que los niños, niñas y adolescentes cuentan con un interés superior para su correcto desarrollo y eso incluye que se satisfagan sus necesidades en todos los aspectos puesto que hay que precautelar el interés superior del menor. Pero no solo es el Estado que debe llevar la responsabilidad, tal como lo plantea Almeida y otros (2020):

Pero no solo por mandato constitucional el estado tiene la obligación de promover el desarrollo de los grupos de atención prioritaria y asegurar el pleno ejercicio de sus derechos atendiendo el principio en análisis también está la familia y sociedad quienes deben atender sus derechos bajo el principio de prevalencia. (p.633)

Se reconoce también en el Código Orgánico de la Niñez y Adolescencia en su artículo 5 de la Ley Reformatoria del Código de la Niñez y Adolescencia; cuales son los obligados subsidiarios en caso de que los padres se encuentren ausentes, tengan impedimento, discapacidad o insuficientes recursos; dichos obligados subsidiarios ya se los menciono con anterioridad, ahora bien entrando en materia que nos compete, bajo esta premisa de "obligados subsidiarios" surgen ciertas inquietudes muy importantes que son eje principal del presente artículo científico, tales como: ¿Qué sucede cuando no existen titulares principales en la obligación alimentaria y tampoco existen obligados subsidiarios? ¿Dónde queda el interés superior del menor en cuanto a las pensiones alimenticias como medio para su desarrollo integral? ¿Cuál es la obligación del Estado en este caso? 
Si bien es cierto la obligación subsidiaria en Ecuador es el vínculo parento-filial que proviene del derecho de familia que nace a partir de que los padres se encuentran imposibilitados a contribuir con la prestación de alimentos y por ende los parientes en cuarto de consanguinidad son llamados por la ley a contribuir con el pago de las pensiones alimenticias a favor del menor, inclusive a favor de menor discapacitados; bebe tener en cuenta y no permitir que por cubrir el derecho del menor se vulnere derechos de los obligados subsidiarios.

La legislación ecuatoriana reconoce el interés superior de los menores como también la obligación de los titulares principales de proporcionar alimentos y a falta de estos sus subsidiarios, en este caso a la no existencia de los mencionados anteriormente, la única opción que queda es la obligación del Estado para con los menores que se han quedado vulnerables y sin ninguna ayuda para su correcto desarrollo como ser humano, tales como salud, educación, alimentación, entre otros. Tanto el Estado como internacionalmente se muestra el interés que se brinda a los estudios sobre pensiones alimenticias e interés superior del niño, "...el tribunal internacionalmente competente para incoar la pretensión alimenticia, habrá que determinar la ley aplicable, que no sólo interesa a fin de conocer la viabilidad y extensión del derecho, sino también de fijar la cantidad que le pudiera corresponder" (Soto, 2016, p. 309).

Desde los planteamientos anteriores el objetivo de la investigación es analizar jurídicamente sobre la responsabilidad del Estado ecuatoriano como obligado subsidiario en los juicios de fijación de pensión alimentaria, a efectos de no vulnerar los derechos de los niños.

\section{MÉTODO}

La investigación se basó en el enfoque cuantitativo de tipo descriptiva de campo no experimental, teniendo como muestra poblacional 30 funcionarios judiciales de la Unidad Judicial de la Mujer, Familia, Niñez y Adolescencia del Cantón Santo Domingo Ecuador, se procedió a aplicar la encuesta conformada por un cuestionario de cinco preguntas, el cual fue validado por el juicio de expertos, calculándose el coeficiente Alfa de Cronbach en razón de prueba piloto, cuyo resultado fue de 0,86 siendo 
considerado confiable para su aplicación, en tal sentido, los datos compilados fueron procesados a través de la estadística descriptiva. Se eligió como técnica el análisis documental y la encuesta cuyos resultados obtenidos permitieron establecer las conclusiones sobre el tema. (Riofrio, 2015).

\section{RESULTADOS}

A continuación, se muestran los resultados obtenidos de la encuesta, de acuerdo las interrogantes formuladas.

\section{Tabla 1.}

Obligados subsidiarios en materia de alimentos.

\begin{tabular}{|l|c|c|}
\hline \multicolumn{1}{|c|}{ VARIABLE } & FRECUENCIA & $\%$ \\
\hline SI & 9 & $30 \%$ \\
\hline NO & 21 & $70 \%$ \\
\hline Total & 30 & $100 \%$ \\
\hline
\end{tabular}

Fuente: Resultados obtenidos del instrumento aplicado a los funcionarios judiciales. Autora (2020).

El principal elemento de la pregunta era saber si los funcionarios judiciales encuestados saben cuáles son los obligados subsidiarios en materia de alimentos, de las 30 encuestas hubo una respuesta positiva del $93 \%$, mientras que el $7 \%$ no conoce cuales son los obligados subsidiarios que establece el Código de la Niñez y Adolescencia. 


\section{Tabla 2.}

En la actual norma vigente diferencia entre objeto obligado principal y subsidiario.

\begin{tabular}{|l|c|c|}
\hline VARIABLE & FRECUENCIA & $\%$ \\
\hline SI & 28 & $93 \%$ \\
\hline NO & 2 & $7 \%$ \\
\hline Total & 30 & $100 \%$ \\
\hline
\end{tabular}

Fuente: Resultados obtenidos del instrumento aplicado a los funcionarios judiciales. Autora (2020).

La segunda pregunta iba encaminada con el fin de establecer si los entrevistados conocían si en la actual norma vigente hay diferencias entre obligado principal y obligado subsidiario, lo cual dio un resultado de que el $30 \%$ de personas encuestadas considera que, si hay diferencias entre el obligado principal con el obligado subsidiario, mientras que el $70 \%$ de personas considera que no existen diferencias entre los obligados subsidiarios y obligados principales en materia de alimentos.

\section{Tabla 3.}

Creen que se afecta al interés superior del niño.

\begin{tabular}{|l|c|c|}
\hline \multicolumn{1}{|c|}{ VARIABLE } & FRECUENCIA & $\%$ \\
\hline SI & 30 & $100 \%$ \\
\hline NO & 0 & $0 \%$ \\
\hline Total & 30 & $100 \%$ \\
\hline
\end{tabular}

Fuente: Resultados obtenidos del instrumento aplicado a los funcionarios judiciales. Autora (2020). 
La tercera interrogante trataba sobre si creen que se afecta al interés superior del niño el hecho de que existan niños, niñas y adolescentes que no puedan percibir su derecho de alimentos, sobre todo cuando se trata de un derecho constitucional, para lo cual los funcionarios que fueron encuestados tuvieron unidad de criterios; el 100\% de los encuestados coincidieron de que si se vulnera el interés superior del niño el hecho de que no puedan percibir ese derecho.

\section{Tabla 4.}

El estado debería ser subsidiario por excepción.

\begin{tabular}{|l|c|c|}
\hline VARIABLE & FRECUENCIA & $\%$ \\
\hline SI & 5 & $29 \%$ \\
\hline NO & 25 & $71 \%$ \\
\hline Total & 30 & $100 \%$ \\
\hline
\end{tabular}

Fuente: Resultados obtenidos del instrumento aplicado a los funcionarios judiciales. Autora (2020).

Aquí se plateó en la cuarta pregunta conocer si los encuestados consideran que el Estado debería ser subsidiario por excepción en casos del derecho de alimentos. Del $100 \%$ un $29 \%$ considera que el Estado no debería ser un subsidiario en materia de derecho de alimentos, mientras que un $71 \%$ considera que el Estado si debería ser obligado subsidiario en razón del interés superior del niño.

\section{Tabla 5.}

La Asamblea Nacional debería reformar la Constitución.

\begin{tabular}{|l|c|c|}
\hline VARIABLE & FRECUENCIA & $\%$ \\
\hline SI & 29 & $97 \%$ \\
\hline NO & 1 & $3 \%$ \\
\hline Total & 30 & $100 \%$ \\
\hline
\end{tabular}

Fuente: Resultados obtenidos del instrumento aplicado a los funcionarios judiciales. Autora (2020). 
Se realizó la siguiente petición en relación a los funcionarios judiciales consideran que la Asamblea Nacional debería reformar la Constitución con el fin de que el Estado asuma su rol de obligado subsidiario en materia de derecho de alimentos cuando existan niños/as y adolescentes que no puedan recibir este derecho puesto que no un familiar paterno o materna, dependiendo el caso, que le pueda proveer este derecho, el resultado fue si para el $97 \%$ y no para el $3 \%$.

\section{DISCUSIÓN}

Los derechos de los niños, niñas y adolescentes son reconocidos por primera vez en la Declaración de Ginebra aprobada en 1924 por la Organización de Naciones Unidas, este es el primer texto histórico reconoce la existencia de derechos específicos para las niños, niñas y adolescentes, además establece la responsabilidad de las personas adultas sobre sus bienes. En 1959 aparece la Declaración de los Derechos del Niño de las Naciones Unidad, pero no fue hasta 1979 cuando se celebró el año Internacional del Niño que la Comisión de Derecho Internacional inicio el proceso de redacción de las normas de la Convención Internacional de los Derechos del niño.

A nivel internacional, la Convención tuvo aceptación por parte de los países desde su origen, 61 Estados fueron parte con la ratificación de 20 países en 1990. Gracias a ello, la Conferencia Mundial de Derechos Humanos convocada por la Organización de Naciones Unidas reunida en Viena en 1993 dio directrices a favor de los niños, como la no discriminación, el interés superior del niño, el derecho a los alimentos, a ser considerados primordiales en actividades que conciernen a la infancia, entre otros. El articulo innumerado 3 del Código de la Niñez y Adolescencia establece las características del derecho a los alimentos de la siguiente manera: "es intransferible, intransmisible, irrenunciable, imprescriptible, inembargable $y$ no admite compensación, ni reembolso de lo pagado" (Código de la Niñez y Adolescencia, 2017). Y es así, porque este derecho no es del padre o la madre, es el derecho que los niños tienen con el fin de tener una vida digna.

Por ello, la obligación de la prestación de alimentos les corresponde como titulares principales a los padres del alimentado, aun en los casos de limitación, suspensión o 
privación de la patria potestad así que en caso de audiencia, impedimento, insuficiencia de recursos o discapacidad de las obligados principales, debidamente comprobado por quien lo alega se ordenara que la prestación de alimentos sea cubierta por uno de los obligados subsidiarios, en atención a su capacidad económica siempre y cuando no se encuentre con ningún tipo de discapacidad.

En lo personal creo que no se puede llamar "obligado subsidiario" puesto que no se ha firmado ningún documento por medio del cual den su aval o garantía en caso de incumplimiento o ausencia del obligado principal; característica principal para que se establezca la figura jurídica de obligación subsidiario, ahora bien, en materia constitucional y por el interés superior del niño que es el eje principal de una sociedad tiene lógica que se establezca una pensión de alimentos para los menores de edad. Lo grave es cuando ni siquiera los obligados subsidiarios tienen los recursos económicos para proveer esa pensión alimenticia, o en el peor de los casos no hay ningún familiar del obligado principal, de los que establece el Código de la Niñez y Adolescencia para cubrir esa necesidad, el alimentado se quedaría sin poder percibir ese derecho, por tal razón seria acucioso que el Estado como principal garantista del derecho de los niños asuma ese rol subsidiario para de esa formar lograr saciar las necesidades que tenga el alimentado, y de esa forma precautelar sus derechos, tales como el derecho a la educación, el derecho al acceso a la misma, esto es transporte, derecho alimentos, a la salud sea cubierto en parte por el Estado. El responsable subsidiario solo ha de responder del deber impuesto al responsable principal si este no lo cumple, no lo puede cumplir, o sencillamente no existe. Así, el acreedor de la obligación primero habrá que dirigirse contra aquel sujeto que sea responsable principal y solo si esta falta, actuar contra el responsable subsidiario, que habrá de responder por el total, sin perjuicio de que más tarde pueda repetir la obligación contra el principal. 


\section{CONCLUSIONES}

Haciendo un análisis crítico y jurídico en el artículo científico se logró llegar a las siguientes conclusiones de acuerdo a la información obtenida mediante encuestas, oficios a instituciones públicas y sobre todo la observación científica mediante estudios bibliográficos más los métodos de investigación aplicados como el empírico y teórico. En la actual norma vigente casi no existen diferencias entre los obligados principales con los obligados subsidiarios en materia de alimentos, lo único que los diferencia es que el obligado subsidiario no será inmune a la prisión por no pagar las pensiones alimenticias, pero eso no quiere decir que por no pagar ahí queda la deuda, la deuda se sigue generando mes a mes en el Sistema Único de Pensiones Alimenticias (SUPA) y si esa persona no quiere tener futuros problemas si algún día quiere trabajar tendrá que estar al día.

En este sentido en la situación de jurídica del país debe atravesar por cambios y reformas específicamente la Constitución de la República y el Código de la Niñez y Adolescencia tipificando al Estado como subsidiario por excepción en pensiones alimenticias, amparados en el interés superior del menor en razón de que no puede ser vulnerado sus derechos constitucionales.

El génesis del problema está en que no existe norma vigente legal especifica del tema tratado y por otra parte los niños, niñas y adolescentes carecen o no cuentan con subsidiarios en razón de pago de pensiones alimenticias, así se ha determinado que, al desarrollar el presente artículo científico, el Estado no está cumpliendo con el interés superior del niño en lo referente al tema desarrollado y es de gran importancia en la actualidad ecuatoriana.

\section{FINANCIAMIENTO}

No monetario.

\section{AGRADECIMIENTO}

A la Universidad Regional Autónoma de Los Andes, Santo Domingo; por motivar el desarrollo de la Investigación. 


\section{REFERENCIAS CONSULTADAS}

Almeida-Toral, P.; Erazo-Álvarez, J.; Ormaza-Ávila, D.; Narváez-Zurita, C. (2020). La aplicación de los derechos humanos en el interés superior del niño. [The application of human rights in the best interests of the child]. IUSTITIA SOCIALIS, 8(5),624-644.

Asamblea Nacional Constituyente de la República del Ecuador, (2008). Constitución de la República del Ecuador. [Constitution of the Republic of Ecuador]. Montecristi. Registro Oficial 449 de 20-oct-2008. Recuperado de https://n9.cl/sia

Asamblea Nacional del Ecuador. (2009). Ley Reformatoria al Código de la Niñez y Adolescencia. [Reform Law to the Childhood and Adolescence Code] Quito: Asamblea Nacional del Ecuador. Obtenido de https://n9.cl/ul23i

Asamblea Nacional del Ecuador. (2015). Código Orgánico General de Procesos. Apremios. [General Organic Code of Processes]. Quito, Pichincha, Ecuador: Registro oficial No. 506.

Cedeño Floril, M.P. (2019). Violencia intrafamiliar: mediación condicionada al tratamiento remedial. [Domestic violence: mediation conditional on remedial treatment]. Universidad y Sociedad. 11(1).193-200. Recuperado de https://n9.cl/ta1v4

Congreso Nacional del Ecuador. (2003). Código de la Niñez y Adolescencia. Derecho de alimentos. [Code of Childhood and Adolescents. Food law]. Quito, Pichincha, Ecuador: Registro oficial No. 737.C

Congreso Nacional del Ecuador. (1970). Código Civil. Título preliminar. [Civil Code] Quito, Pichincha, Ecuador: Registro Oficial No. 104.

Naciones Unidas. (2006). Convención de Derechos del Niños de las Naciones Unidas. [United Nations Convention on the Rights of the Child]. Madrid: UNICEF.

Organización de Naciones Unidas. (1989). Convención sobre los derechos del Niño. Interés superior del niño. [Invention on the rights of the Child. Child's best interest] San Francisco, Estados Unidos: ONU.

Riofrío, J. (2015). La selección del método en la investigación jurídica. [Selection of the method in legal research]. Educación y Derecho. Recuperado de: https://n9.cl/ju7p 
lustitia Socialis. Revista Arbitrada de Ciencias Jurídicas.

Año V. Vol. V. №3. Edición Especial. 2020-III:

Universidad Regional Autónoma de los Andes

Hecho el depósito de Ley: FA2016000064

ISSN: 2542-3371

FUNDACIÓN KOINONIA (F.K). Santa Ana de Coro, Venezuela

Julián Rodolfo Santillán-Andrade; Jaritza Andreina Aguirre-Aguilar

Soto, M. (2016). Prestación alimenticia en las relaciones hispano Argentinas. [Food provision in Hispanic-Argentine relations] Boletín mexicano de derecho comparado, 49(145). Recuperado de: https://n9.cl/jx55.

(C2020 por los autores. Este artículo es de acceso abierto y distribuido según los términos y condiciones de la licencia Creative Commons Atribución-NoComercial-Compartirlgual 4.0 Internacional (CC BY-NC-SA 4.0) (https://creativecommons.org/licenses/by-nc-sa/4.0/). 